\title{
THREE CLOSED FORMS FOR CONVOLVED FIBONACCI NUMBERS
}

\author{
FENG QI
}

Dedicated to people facing and battling COVID-19

\begin{abstract}
In the paper, by virtue of the Faà di Bruno formula and several properties of the Bell polynomials of the second kind, the author computes higher order derivatives of the generating function of convolved Fibonacci numbers and, consequently, derives three closed forms for convolved Fibonacci numbers in terms of the falling and rising factorials, the Lah numbers, the signed Stirling numbers of the first kind, and the golden ratio.
\end{abstract}

\section{Motivation And MAin RESUlts}

The well-known Fibonacci numbers

$$
F_{n}=\frac{1}{\sqrt{5}}\left[\left(\frac{1+\sqrt{5}}{2}\right)^{n}-\left(\frac{1-\sqrt{5}}{2}\right)^{n}\right], \quad n \in \mathbb{N}
$$

form a sequence of integers and satisfy the linear recurrence relation $F_{n}=F_{n-1}+$ $F_{n-2}$ for $n \geq 3$. The first eight Fibonacci numbers $F_{n}$ for $1 \leq n \leq 8$ are $1,1,2,3,5,8,13,21$. We can generate all the Fibonacci numbers $F_{n}$ for $n \geq 1$ by

$$
\frac{1}{1-t-t^{2}}=\sum_{n=0}^{\infty} F_{n+1} t^{n}=1+t+2 t^{2}+3 t^{3}+5 t^{4}+8 t^{5}+\cdots, \quad|t|<\frac{\sqrt{5}-1}{2} .
$$

We can find the definition of convolved Fibonacci numbers $\mathcal{F}_{n}(x)$

$$
F(t, x)=\left(\frac{1}{1-t-t^{2}}\right)^{x}=\sum_{n=0}^{\infty} \mathcal{F}_{n}(x) \frac{t^{n}}{n !}, \quad x \in \mathbb{R}
$$

in the papers 2 , 3, 5, 12. It is obvious that $\mathcal{F}_{n}(1)=n ! F_{n+1}$ for $n \geq 0$.

Kim and his three coauthors [11, Theorem 6] proved in an elementary fashion by induction and recursion that the family of differential equations

$$
\frac{\partial^{n} F(t, x)}{\partial t^{n}}=\left[\sum_{i=0}^{\left\lfloor\frac{n+1}{2}\right\rfloor} a_{i}(n)(x)_{n-i} \frac{(1+2 t)^{n-2 i}}{\left(1-t-t^{2}\right)^{n-i}}\right] F(t, x)
$$

2010 Mathematics Subject Classification. Primary 11B39; Secondary 11B65, 11B73, 11B83, 26A06, 26A09.

Key words and phrases. closed form; convolved Fibonacci number; Faà di Bruno formula; Bell polynomial of the second kind; higher order derivative; generating function; falling factorial; rising factorial; Lah number; signed Stirling number of the first kind; golden ratio.

This paper was typeset using $\mathcal{A}_{\mathcal{M}} \mathcal{S}$ - $\mathrm{L}_{\mathrm{A}} \mathrm{T} \mathrm{E}$. 
have a solution $F(t, x)=\left(\frac{1}{1-t-t^{2}}\right)^{x}$, where $\lfloor x\rfloor$ is the floor function whose value equals the largest integer less than or equal to $x$, the quantity

$$
(x)_{n}=\prod_{\ell=0}^{n-1}(x+\ell)= \begin{cases}x(x+1) \cdots(x+n-1), & n \geq 1 \\ 1, & n=0\end{cases}
$$

denotes the rising factorial, and

$$
a_{i}(n)= \begin{cases}1, & i=0 ; \\ 2^{i} \sum_{k_{i}=1}^{n-2 i+1} \sum_{k_{i-1}=1}^{k_{i}+1} \ldots \sum_{k_{1}=1}^{k_{2}+1} \prod_{\ell=1}^{i} k_{\ell}, & 1 \leq i \leq\left\lfloor\frac{n}{2}\right\rfloor \\ 0, & i=\frac{n+1}{2}\end{cases}
$$

for all $n \in \mathbb{N}$. Consequently, the authors [11, Corollary 8] derived that

$$
\mathcal{F}_{n}(x)=\sum_{i=0}^{\left\lfloor\frac{n+1}{2}\right\rfloor} a_{i}(n)(x)_{n-i}
$$

It is clear that computing, remembering, and understanding the above quantity $a_{i}(n)$ by hands and brains are not an easy thing. Generally speaking, beauty, simplicity, symmetry, and recursion are the best and the hope to find solutions in mathematics.

In this paper, our aims are to supply three alternative families of differential equations for the generating function $F(t, x)$ of convolved Fibonacci numbers $\mathcal{F}_{n}(x)$ in the form of higher order derivatives and to provide three alternative closed forms for convolved Fibonacci numbers $\mathcal{F}_{n}(x)$ in terms of the falling factorials $\langle x\rangle_{k}$, the rising factorials $(x)_{k}$, the Lah numbers $L(n, k)$, the signed Stirling numbers of the first kind $s(n, k)$, and the golden ratio $\frac{\sqrt{5}+1}{2}$.

Our main results can be stated as the following theorems.

Theorem 1. The nth partial derivative with respect to $t$ of the generating function $F(t, x)$ can be computed by

$$
\frac{\partial^{n} F(t, x)}{\partial t^{n}}=\left[\frac{1}{\left(\frac{\sqrt{5}-1}{2}-t\right)^{n}} \sum_{k=0}^{n}(-1)^{k}\left(\begin{array}{l}
n \\
k
\end{array}\right)(x)_{k}(x)_{n-k}\left(\frac{\frac{\sqrt{5}-1}{2}-t}{t+\frac{\sqrt{5}+1}{2}}\right)^{k}\right] F(t, x) .
$$

Consequently, convolved Fibonacci numbers $\mathcal{F}_{n}(x)$ for $n \geq 0$ can be expressed as

$$
\mathcal{F}_{n}(x)=\frac{1}{\left(\frac{\sqrt{5}-1}{2}\right)^{n}} \sum_{k=0}^{n}(-1)^{k}\left(\begin{array}{l}
n \\
k
\end{array}\right)\left(\frac{\sqrt{5}-1}{\sqrt{5}+1}\right)^{k}(x)_{k}(x)_{n-k} .
$$

Theorem 2. The $n$th partial derivative with respect to $t$ of the generating function $F(t, x)$ can be computed by

$$
\frac{\partial^{n} F(t, x)}{\partial t^{n}}=\left[\sum_{k=0}^{n}(-1)^{k} x^{k} \sum_{r+s=k} \sum_{\ell+m=n}\left(\begin{array}{l}
n \\
\ell
\end{array}\right) \frac{s(\ell, r) s(m, s)}{\left(t+\frac{\sqrt{5}+1}{2}\right)^{\ell}\left(t-\frac{\sqrt{5}-1}{2}\right)^{m}}\right] F(t, x),
$$


where $s(n, k)$ are the signed Stirling numbers of the first kind which can be generated by

$$
\frac{(\ln (1+x))^{k}}{k !}=\sum_{n=k}^{\infty} s(n, k) \frac{x^{n}}{n !}, \quad|x|<1 .
$$

Consequently, convolved Fibonacci numbers $\mathcal{F}_{n}(x)$ for $n \geq 0$ can be expressed as

$$
\mathcal{F}_{n}(x)=\sum_{k=0}^{n}(-1)^{k} x^{k} \sum_{r+s=k} \sum_{\ell+m=n}(-1)^{m}\left(\begin{array}{l}
n \\
\ell
\end{array}\right) s(\ell, r) s(m, s)\left(\frac{\sqrt{5}+1}{2}\right)^{m-\ell} .
$$

Theorem 3. The nth partial derivative with respect to $t$ of the generating function $F(t, x)$ can be computed by

$$
\begin{aligned}
\frac{\partial^{n} F(t, x)}{\partial t^{n}}=\left[\sum_{k=0}^{n} \frac{\langle x\rangle_{k}}{(\sqrt{5})^{k}}\right. & \sum_{r+s=k} \sum_{\ell+m=n}(-1)^{r}\left(\begin{array}{l}
n \\
\ell
\end{array}\right) L(\ell, r) L(m, s) \\
& \left.\times\left(t+\frac{\sqrt{5}+1}{2}\right)^{s-\ell}\left(t-\frac{\sqrt{5}-1}{2}\right)^{r-m}\right] F(t, x),
\end{aligned}
$$

where

are the Lah numbers and

$$
L(n, k)=\left(\begin{array}{l}
n-1 \\
k-1
\end{array}\right) \frac{n !}{k !}, \quad n \geq k \geq 0
$$

$$
\langle x\rangle_{n}=\prod_{k=0}^{n-1}(x-k)= \begin{cases}x(x-1) \cdots(x-n+1), & n \geq 1 \\ 1, & n=0\end{cases}
$$

is the falling factorial. Consequently, convolved Fibonacci numbers $\mathcal{F}_{n}(x)$ for $n \geq 0$ can be expressed as

$$
\mathcal{F}_{n}(x)=\sum_{k=0}^{n} \frac{\langle x\rangle_{k}}{(\sqrt{5})^{k}} \sum_{r+s=k} \sum_{\ell+m=n}(-1)^{m}\left(\begin{array}{l}
n \\
\ell
\end{array}\right) L(\ell, r) L(m, s)\left(\frac{\sqrt{5}+1}{2}\right)^{s+m-r-\ell} .
$$

\section{LEMMAS}

In order to verify our main results in Theorems 1 to 3 , we need some notions and lemmas below.

In combinatorial mathematics, the Bell polynomials of the second kind $\mathrm{B}_{n, k}$ are defined by

$$
\mathrm{B}_{n, k}\left(x_{1}, x_{2}, \ldots, x_{n-k+1}\right)=\sum_{\substack{1 \leq i \leq n-k+1 \\ \ell_{i} \in\{0\} \cup \mathbb{N} \\ \sum_{i=1}^{n-k+1} i \ell_{i}=n \\ \sum_{i=1}^{n-k+1} \ell_{i}=k}} \frac{n !}{\prod_{i=1}^{n-k+1} \ell_{i} !} \prod_{i=1}^{n-k+1}\left(\frac{x_{i}}{i !}\right)^{\ell_{i}}
$$

for $n \geq k \geq 0$. See [4, p. 134, Theorem A]. In terms of the Bell polynomials of the second kind $\mathrm{B}_{n, k}$, the Faà di Bruno formula for computing higher order derivatives of composite functions is described in [4, p. 139, Theorem C] by

$$
\frac{\mathrm{d}^{n}}{\mathrm{~d} x^{n}} f \circ g(x)=\sum_{k=0}^{n} f^{(k)}(g(x)) \mathrm{B}_{n, k}\left(g^{\prime}(x), g^{\prime \prime}(x), \ldots, g^{(n-k+1)}(x)\right) .
$$


The Bell polynomials of the second kind $\mathrm{B}_{n, k}$ have the following properties.

Lemma 1 ([1, Example 2.6] and [4, p. 136, Eq. [3n]]). The Bell polynomials of the second kind $\mathrm{B}_{n, k}$ satisfy

$$
\begin{aligned}
\mathrm{B}_{n, k} & \left(x_{1}+y_{1}, x_{2}+y_{2}, \ldots, x_{n-k+1}+y_{n-k+1}\right) \\
& =\sum_{r+s=k} \sum_{\ell+m=n}\left(\begin{array}{l}
n \\
\ell
\end{array}\right) \mathrm{B}_{\ell, r}\left(x_{1}, x_{2}, \ldots, x_{\ell-r+1}\right) \mathrm{B}_{m, s}\left(y_{1}, y_{2}, \ldots, y_{m-s+1}\right) .
\end{aligned}
$$

Lemma 2 ([4, p. 135]). For $n \geq k \geq 0$, we have

$$
\mathrm{B}_{n, k}\left(a b x_{1}, a b^{2} x_{2}, \ldots, a b^{n-k+1} x_{n-k+1}\right)=a^{k} b^{n} \mathrm{~B}_{n, k}\left(x_{1}, x_{2}, \ldots, x_{n-k+1}\right),
$$

where $a$ and $b$ are any complex numbers.

Lemma 3 (4, p. 135, Theorem B] and [18, p. 27, eq. (3.1)]). For $n \geq k \geq 0$, we have

$$
\mathrm{B}_{n, k}(1 !, 2 !, \ldots,(n-k+1) !)=\left(\begin{array}{c}
n-1 \\
k-1
\end{array}\right) \frac{n !}{k !}=L(n, k)
$$

and

$$
\mathrm{B}_{n, k}(0 !, 1 !, 2 !, \ldots,(n-k) !)=(-1)^{n-k} s(n, k) .
$$

\section{Proofs of MAIN Results}

Now we are in a position to prove our main results in Theorems 1 to 3. Proof of Theorem 1. It is clear that the function $\frac{1}{1-t-t^{2}}$ can be written as

$$
\frac{1}{1-t-t^{2}}=\frac{1}{\left(t+\frac{\sqrt{5}+1}{2}\right)\left(\frac{\sqrt{5}-1}{2}-t\right)} .
$$

Hence, it follows that

$$
F(t, x)=\left(\frac{1}{1-t-t^{2}}\right)^{x}=\frac{1}{\left(t+\frac{\sqrt{5}+1}{2}\right)^{x}\left(\frac{\sqrt{5}-1}{2}-t\right)^{x}}
$$

and

$$
\begin{aligned}
\frac{\partial^{n} F(t, x)}{\partial t^{n}} & =\sum_{k=0}^{n}\left(\begin{array}{l}
n \\
k
\end{array}\right) \frac{\partial^{k}}{\partial t^{k}}\left[\frac{1}{\left(t+\frac{\sqrt{5}+1}{2}\right)^{x}}\right] \frac{\partial^{n-k}}{\partial t^{n-k}}\left[\frac{1}{\left(\frac{\sqrt{5}-1}{2}-t\right)^{x}}\right] \\
& =\sum_{k=0}^{n}\left(\begin{array}{l}
n \\
k
\end{array}\right) \frac{(-1)^{k}(x)_{k}}{\left(t+\frac{\sqrt{5}+1}{2}\right)^{x+k}} \frac{(x)_{n-k}}{\left(\frac{\sqrt{5}-1}{2}-t\right)^{x+n-k}} \\
& =\left(\frac{1}{1-t-t^{2}}\right)^{x} \frac{1}{\left(\frac{\sqrt{5}-1}{2}-t\right)^{n}} \sum_{k=0}^{n}(-1)^{k}\left(\begin{array}{l}
n \\
k
\end{array}\right)\left(\frac{\frac{\sqrt{5}-1}{2}-t}{t+\frac{\sqrt{5}+1}{2}}\right)^{k}(x)_{k}(x)_{n-k} \\
& \rightarrow \frac{1}{\left(\frac{\sqrt{5}-1}{2}\right)^{n}} \sum_{k=0}^{n}(-1)^{k}\left(\begin{array}{l}
n \\
k
\end{array}\right)\left(\frac{\sqrt{5}-1}{\sqrt{5}+1}\right)^{k}(x)_{k}(x)_{n-k}
\end{aligned}
$$

as $t \rightarrow 0$. The proof of Theorem 1 is complete. 
Proof of Theorem 2. It is clear that we can write

$$
\left(\frac{1}{1-t-t^{2}}\right)^{x}=e^{-x \ln \left(1-t-t^{2}\right)}
$$

Applying the Faà di Bruno formula (2.1) to $f(u)=e^{ \pm u}$ and

$$
u=g(t)=\mp x \ln \left(1-t-t^{2}\right)=\mp x\left[\ln \left(t+\frac{\sqrt{5}+1}{2}\right)+\ln \left(\frac{\sqrt{5}-1}{2}-t\right)\right]
$$

and employing the formulas $(2.2),(2.3)$, and $(2.5)$ yield

$$
\begin{aligned}
& \frac{\mathrm{d}^{n}}{\mathrm{~d} t^{n}}\left[\left(\frac{1}{1-t-t^{2}}\right)^{x}\right]=\sum_{k=0}^{n} \frac{\mathrm{d}^{k}\left(e^{ \pm u}\right)}{\mathrm{d} u^{k}} \mathrm{~B}_{n, k}\left(\mp x\left[\frac{0 !}{t+\frac{\sqrt{5}+1}{2}}+\frac{0 !}{t-\frac{\sqrt{5}-1}{2}}\right],\right. \\
& \mp x\left[\frac{(-1)^{1} 1 !}{\left(t+\frac{\sqrt{5}+1}{2}\right)^{2}}+\frac{(-1)^{1} 1 !}{\left(t-\frac{\sqrt{5}-1}{2}\right)^{2}}\right], \mp x\left[\frac{(-1)^{2} 2 !}{\left(t+\frac{\sqrt{5}+1}{2}\right)^{3}}+\frac{(-1)^{2} 2 !}{\left(t-\frac{\sqrt{5}-1}{2}\right)^{3}}\right] \text {, } \\
& \left.\ldots, \mp x\left[\frac{(-1)^{n-k}(n-k) !}{\left(t+\frac{\sqrt{5}+1}{2}\right)^{n-k+1}}+\frac{(-1)^{n-k}(n-k) !}{\left(t-\frac{\sqrt{5}-1}{2}\right)^{n-k+1}}\right]\right) \\
& =\sum_{k=0}^{n}( \pm 1)^{k} e^{ \pm u}(\mp x)^{k}(-1)^{n+k} \mathrm{~B}_{n, k}\left(\frac{0 !}{t+\frac{\sqrt{5}+1}{2}}+\frac{0 !}{t-\frac{\sqrt{5}-1}{2}}, \frac{1 !}{\left(t+\frac{\sqrt{5}+1}{2}\right)^{2}}\right. \\
& +\frac{1 !}{\left(t-\frac{\sqrt{5}-1}{2}\right)^{2}}, \frac{2 !}{\left(t+\frac{\sqrt{5}+1}{2}\right)^{3}}+\frac{2 !}{\left(t-\frac{\sqrt{5}-1}{2}\right)^{3}}, \ldots, \\
& \left.\frac{(n-k) !}{\left(t+\frac{\sqrt{5}+1}{2}\right)^{n-k+1}}+\frac{(n-k) !}{\left(t-\frac{\sqrt{5}-1}{2}\right)^{n-k+1}}\right) \\
& =(-1)^{n}\left(\frac{1}{1-t-t^{2}}\right)^{x} \sum_{k=0}^{n} x^{k} \sum_{r+s=k} \sum_{\ell+m=n}\left(\begin{array}{l}
n \\
\ell
\end{array}\right) \mathrm{B}_{\ell, r}\left(\frac{0 !}{t+\frac{\sqrt{5}+1}{2}}\right. \text {, } \\
& \left.\frac{1 !}{\left(t+\frac{\sqrt{5}+1}{2}\right)^{2}}, \frac{2 !}{\left(t+\frac{\sqrt{5}+1}{2}\right)^{3}}, \ldots, \frac{(\ell-r) !}{\left(t+\frac{\sqrt{5}+1}{2}\right)^{\ell-r+1}}\right) \mathrm{B}_{m, s}\left(\frac{0 !}{t-\frac{\sqrt{5}-1}{2}},\right. \\
& \left.\frac{1 !}{\left(t-\frac{\sqrt{5}-1}{2}\right)^{2}}, \frac{2 !}{\left(t-\frac{\sqrt{5}-1}{2}\right)^{3}}, \ldots, \frac{(m-s) !}{\left(t-\frac{\sqrt{5}-1}{2}\right)^{m-s+1}}\right) \\
& =(-1)^{n}\left(\frac{1}{1-t-t^{2}}\right)^{x} \sum_{k=0}^{n} x^{k} \sum_{r+s=k} \sum_{\ell+m=n}\left(\begin{array}{l}
n \\
\ell
\end{array}\right) \\
& \times \frac{\mathrm{B}_{\ell, r}(0 !, 1 !, \ldots,(\ell-r) !)}{\left(t+\frac{\sqrt{5}+1}{2}\right)^{\ell}} \frac{\mathrm{B}_{m, s}(0 !, 1 !, \ldots,(m-s) !)}{\left(t-\frac{\sqrt{5}-1}{2}\right)^{m}} \\
& =(-1)^{n}\left(\frac{1}{1-t-t^{2}}\right)^{x} \sum_{k=0}^{n} x^{k} \sum_{r+s=k} \sum_{\ell+m=n}\left(\begin{array}{l}
n \\
\ell
\end{array}\right) \frac{(-1)^{\ell-r} s(\ell, r)}{\left(t+\frac{\sqrt{5}+1}{2}\right)^{\ell}} \frac{(-1)^{m-s} s(m, s)}{\left(t-\frac{\sqrt{5}-1}{2}\right)^{m}} \\
& =\left(\frac{1}{1-t-t^{2}}\right)^{x} \sum_{k=0}^{n}(-1)^{k} x^{k} \sum_{r+s=k} \sum_{\ell+m=n}\left(\begin{array}{l}
n \\
\ell
\end{array}\right) \frac{s(\ell, r) s(m, s)}{\left(t+\frac{\sqrt{5}+1}{2}\right)^{\ell}\left(t-\frac{\sqrt{5}-1}{2}\right)^{m}}
\end{aligned}
$$

and, consequently, 


$$
\begin{aligned}
& \mathcal{F}_{n}(x)=\lim _{t \rightarrow 0} \frac{\mathrm{d}^{n}}{\mathrm{~d} t^{n}}\left[\left(\frac{1}{1-t-t^{2}}\right)^{x}\right] \\
&=\sum_{k=0}^{n}(-1)^{k} x^{k} \sum_{r+s=k} \sum_{\ell+m=n}\left(\begin{array}{l}
n \\
\ell
\end{array}\right) \frac{s(\ell, r) s(m, s)}{\left(\frac{\sqrt{5}+1}{2}\right)^{\ell}\left(-\frac{\sqrt{5}-1}{2}\right)^{m}} \\
&=\sum_{k=0}^{n}(-1)^{k} x^{k} \sum_{r+s=k} \sum_{\ell+m=n}(-1)^{m}\left(\begin{array}{l}
n \\
\ell
\end{array}\right) s(\ell, r) s(m, s)\left(\frac{\sqrt{5}+1}{2}\right)^{m}\left(\frac{\sqrt{5}-1}{2}\right)^{\ell} \\
&=\sum_{k=0}^{n}(-1)^{k} x^{k} \sum_{r+s=k} \sum_{\ell+m=n}(-1)^{m}\left(\begin{array}{l}
n \\
\ell
\end{array}\right) s(\ell, r) s(m, s)\left(\frac{\sqrt{5}+1}{2}\right)^{m-\ell} .
\end{aligned}
$$

The proof of Theorem 2 is thus complete.

Proof of Theorem 3 . Let $u=g(t)=\frac{1}{1-t-t^{2}}$. Then, by the Faà di Bruno formula 2.1,

$$
\begin{aligned}
\frac{\mathrm{d}^{n}}{\mathrm{~d} t^{n}}\left[\left(\frac{1}{1-t-t^{2}}\right)^{x}\right] & =\sum_{k=0}^{n} \frac{\mathrm{d}^{k}\left(u^{x}\right)}{\mathrm{d} u^{k}} \mathrm{~B}_{n, k}\left(g^{\prime}(t), g^{\prime \prime}(t), \ldots, g^{(n-k+1)}(t)\right) \\
& =\sum_{k=0}^{n}\langle x\rangle_{k} g^{x-k}(t) \mathrm{B}_{n, k}\left(g^{\prime}(t), g^{\prime \prime}(t), \ldots, g^{(n-k+1)}(t)\right)
\end{aligned}
$$

Since

$$
g(t)=\frac{1}{\sqrt{5}}\left(\frac{1}{t+\frac{\sqrt{5}+1}{2}}-\frac{1}{t-\frac{\sqrt{5}-1}{2}}\right)
$$

and

$$
g^{(k)}(t)=\frac{k !}{\sqrt{5}}\left[\frac{1}{\left(t+\frac{\sqrt{5}+1}{2}\right)^{k+1}}-\frac{1}{\left(t-\frac{\sqrt{5}-1}{2}\right)^{k+1}}\right],
$$

by the formulas $2.2,2.3$, and 2.4, we obtain

$$
\begin{gathered}
\mathrm{B}_{n, k}\left(g^{\prime}(t), g^{\prime \prime}(t), \ldots, g^{(n-k+1)}(t)\right)=\sum_{r+s=k} \sum_{\ell+m=n}\left(\begin{array}{l}
n \\
\ell
\end{array}\right) \mathrm{B}_{\ell, r}\left(\frac{1 !}{\sqrt{5}} \frac{1}{\left(t+\frac{\sqrt{5}+1}{2}\right)^{2}},\right. \\
\left.\frac{2 !}{\sqrt{5}} \frac{1}{\left(t+\frac{\sqrt{5}+1}{2}\right)^{3}}, \ldots, \frac{(\ell-r+1) !}{\sqrt{5}} \frac{1}{\left(t+\frac{\sqrt{5}+1}{2}\right)^{\ell-r+2}}\right) \mathrm{B}_{m, s}\left(-\frac{1 !}{\sqrt{5}} \frac{1}{\left(t-\frac{\sqrt{5}-1}{2}\right)^{2}}\right. \\
\left.\quad-\frac{2 !}{\sqrt{5}} \frac{1}{\left(t-\frac{\sqrt{5}-1}{2}\right)^{3}}, \ldots,-\frac{(m-s+1) !}{\sqrt{5}} \frac{1}{\left(t-\frac{\sqrt{5}-1}{2}\right)^{m-s+2}}\right) \\
=\sum_{r+s=k} \sum_{\ell+m=n}\left(\begin{array}{l}
n \\
\ell
\end{array}\right)\left(\frac{1}{\sqrt{5}}\right)^{r} \frac{1}{\left(t+\frac{\sqrt{5}+1}{2}\right)^{\ell+r}} \mathrm{~B}_{\ell, r}(1 !, 2 !, \ldots,(\ell-r+1) !) \\
\quad \times\left(-\frac{1}{\sqrt{5}}\right)^{s} \frac{1}{\left(t-\frac{\sqrt{5}-1}{2}\right)^{m+s}} \mathrm{~B}_{m, s}(1 !, 2 !, \ldots,(m-s+1) !) \\
=\left(\frac{1}{\sqrt{5}}\right)^{k} \sum_{r+s=k} \sum_{\ell+m=n}(-1)^{s}\left(\begin{array}{l}
n \\
\ell
\end{array}\right) \frac{1}{\left(t+\frac{\sqrt{5}+1}{2}\right)^{\ell+r}} \frac{1}{\left(t-\frac{\sqrt{5}-1}{2}\right)^{m+s}} L(\ell, r) L(m, s) .
\end{gathered}
$$


Therefore, it follows that

$$
\begin{aligned}
& \frac{\mathrm{d}^{n}}{\mathrm{~d} t^{n}}\left[\left(\frac{1}{1-t-t^{2}}\right)^{x}\right]=g^{x}(t) \sum_{k=0}^{n} \frac{\langle x\rangle_{k}}{g^{k}(t)}\left(\frac{1}{\sqrt{5}}\right)^{k} \\
& \times \sum_{r+s=k} \sum_{\ell+m=n}(-1)^{s}\left(\begin{array}{l}
n \\
\ell
\end{array}\right) L(\ell, r) L(m, s) \frac{1}{\left(t+\frac{\sqrt{5}+1}{2}\right)^{\ell+r}} \frac{1}{\left(t-\frac{\sqrt{5}-1}{2}\right)^{m+s}} \\
& =\left(\frac{1}{1-t-t^{2}}\right)^{x} \sum_{k=0}^{n} \frac{\langle x\rangle_{k}}{(\sqrt{5})^{k}}\left(1-t-t^{2}\right)^{k} \\
& \times \sum_{r+s=k} \sum_{\ell+m=n}(-1)^{s}\left(\begin{array}{l}
n \\
\ell
\end{array}\right) L(\ell, r) L(m, s) \frac{1}{\left(t+\frac{\sqrt{5}+1}{2}\right)^{\ell+r}} \frac{1}{\left(t-\frac{\sqrt{5}-1}{2}\right)^{m+s}} \\
& =\left(\frac{1}{1-t-t^{2}}\right)^{x} \sum_{k=0}^{n} \frac{\langle x\rangle_{k}}{(\sqrt{5})^{k}} \sum_{r+s=k} \sum_{\ell+m=n}(-1)^{s+k}\left(\begin{array}{l}
n \\
\ell
\end{array}\right) L(\ell, r) L(m, s) \\
& \times \frac{\left(t-\frac{\sqrt{5}-1}{2}\right)^{k}\left(t+\frac{\sqrt{5}+1}{2}\right)^{k}}{\left(t+\frac{\sqrt{5}+1}{2}\right)^{\ell+r}\left(t-\frac{\sqrt{5}-1}{2}\right)^{m+s}} \\
& =\left(\frac{1}{1-t-t^{2}}\right)^{x} \sum_{k=0}^{n} \frac{\langle x\rangle_{k}}{(\sqrt{5})^{k}} \sum_{r+s=k} \sum_{\ell+m=n}(-1)^{r}\left(\begin{array}{l}
n \\
\ell
\end{array}\right) L(\ell, r) L(m, s) \\
& \times \frac{\left(t+\frac{\sqrt{5}+1}{2}\right)^{s}\left(t-\frac{\sqrt{5}-1}{2}\right)^{r}}{\left(t+\frac{\sqrt{5}+1}{2}\right)^{\ell}\left(t-\frac{\sqrt{5}-1}{2}\right)^{m}} \\
& =\left(\frac{1}{1-t-t^{2}}\right)^{x} \sum_{k=0}^{n} \frac{\langle x\rangle_{k}}{(\sqrt{5})^{k}} \sum_{r+s=k} \sum_{\ell+m=n}(-1)^{r}\left(\begin{array}{l}
n \\
\ell
\end{array}\right) L(\ell, r) L(m, s) \\
& \times\left(t+\frac{\sqrt{5}+1}{2}\right)^{s-\ell}\left(t-\frac{\sqrt{5}-1}{2}\right)^{r-m}
\end{aligned}
$$

and, consequently,

$$
\begin{aligned}
& \mathcal{F}_{n}(x)=\lim _{t \rightarrow 0} \frac{\mathrm{d}^{n}}{\mathrm{~d} t^{n}}\left[\left(\frac{1}{1-t-t^{2}}\right)^{x}\right] \\
& =\sum_{k=0}^{n} \frac{\langle x\rangle_{k}}{(\sqrt{5})^{k}} \sum_{r+s=k} \sum_{\ell+m=n}(-1)^{m}\left(\begin{array}{c}
n \\
\ell
\end{array}\right) L(\ell, r) L(m, s)\left(\frac{\sqrt{5}+1}{2}\right)^{s-\ell}\left(\frac{\sqrt{5}-1}{2}\right)^{r-m} \\
& =\sum_{k=0}^{n} \frac{\langle x\rangle_{k}}{(\sqrt{5})^{k}} \sum_{r+s=k} \sum_{\ell+m=n}(-1)^{m}\left(\begin{array}{c}
n \\
\ell
\end{array}\right) L(\ell, r) L(m, s)\left(\frac{\sqrt{5}+1}{2}\right)^{s+m-r-\ell} .
\end{aligned}
$$

The differential equations in 1.9 and the formula 1.10 are thus verified. The proof of Theorem 3 is complete.

\section{REMARKS}

Finally, we list several remarks on our main results and other things.

Remark 1. Because the differential equations in 1.5$),(1.7)$, and $(1.9)$ and the closed forms (1.6), 1.8), and (1.10) are expressed in terms of the falling factorials $\langle x\rangle_{k}$, the rising factorial $(x)_{k}$, the Lah numbers $L(n, k)$, the signed Stirling numbers of 
the first kind $s(n, k)$, and the golden ratio $\frac{\sqrt{5}+1}{2}$, they and their proofs are simpler, more meaningful, more significant, and more computable than (1.3) and (1.4) and their proofs in [1].

Remark 2. By 1.1 and 3.1, we can conclude that

$$
\begin{aligned}
& \mathrm{B}_{n, k}\left(1 ! F_{2}, 2 ! F_{3}, \ldots,(n-k+1) ! F_{n-k+2}\right) \\
= & \left(\frac{1}{\sqrt{5}}\right)^{k} \sum_{r+s=k} \sum_{\ell+m=n}(-1)^{m}\left(\begin{array}{l}
n \\
\ell
\end{array}\right) \frac{L(\ell, r) L(m, s)}{\left(\frac{\sqrt{5}+1}{2}\right)^{\ell+r}\left(\frac{\sqrt{5}-1}{2}\right)^{m+s}} \\
= & \left(\frac{1}{\sqrt{5}}\right)^{k} \sum_{r+s=k} \sum_{\ell+m=n}(-1)^{m}\left(\begin{array}{l}
n \\
\ell
\end{array}\right) L(\ell, r) L(m, s)\left(\frac{\sqrt{5}+1}{2}\right)^{s+m-r-\ell} .
\end{aligned}
$$

By the way, in the papers [6, 14, 17, 29, 33, 37, 40, 46, 42, 47, 48, 50, 55] and closely-related references therein, there are some new results and applications of special values of the Bell polynomials of the second kind $\mathrm{B}_{n, k}$.

Remark 3. In the papers [8, 10, 49] and closely-related references, there are some new results for the Lah numbers $L(n, k)$. In the papers [13, 18, 19, 21, 22, 30, and references cited therein, there are some new results for the signed Stirling numbers of the first kind $s(n, k)$.

Remark 4. In [29], the authors have discussed the Cauchy product of central Delannoy numbers. We will investigate convolved central Delannoy numbers in a subsequent article.

Remark 5. We can generate the Fibonacci polynomials

$$
F_{n}(s)=\frac{1}{2^{n}} \frac{\left(s+\sqrt{4+s^{2}}\right)^{n}-\left(s-\sqrt{4+s^{2}}\right)^{n}}{\sqrt{4+s^{2}}}, \quad n \in \mathbb{N}
$$

by

$$
\frac{1}{1-s z-z^{2}}=\sum_{n=0}^{\infty} F_{n+1}(s) z^{n}=1+s z+\left(s^{2}+1\right) z^{2}+\left(s^{3}+2 s\right) z^{3}+\cdots
$$

One can define the generalized Fibonacci polynomials $F_{n}(s, t)$ by the initial values

$$
F_{0}(s, t)=0, \quad F_{1}(s, t)=1,
$$

and the recurrence relation

$$
F_{n}(s, t)=s F_{n-1}(s, t)+t F_{n-2}(s, t), \quad n \geq 2 .
$$

It is easy to deduce that

$$
F_{2}(s, t)=s, \quad F_{3}(s, t)=s^{2}+t, \quad F_{4}(s, t)=s^{3}+2 s t, \quad F_{5}(s, t)=s^{4}+3 s^{2} t+t^{2} .
$$

We can generate the generalized Fibonacci polynomials $F_{n}(s, t)$ for $n \in \mathbb{N}$ by

$$
\frac{1}{1-s z-t z^{2}}=\sum_{n=0}^{\infty} F_{n+1}(s, t) z^{n} .
$$

For more information, please refer to 34 and closely-related references therein.

It is clear that $F_{n}(s, 1)=F_{n}(s)$ and $F_{n}(1,1)=F_{n}(1)=F_{n}$ for $n \in \mathbb{N}$. 
Motivated by the definition (1.2) for convolved Fibonacci numbers $\mathcal{F}_{n}(x)$, we can introduce two new notions, convolved Fibonacci polynomials $\mathcal{F}_{n+1}(s ; x)$ and convolved generalized Fibonacci polynomials $\mathcal{F}_{n+1}(s, t ; x)$, by

$$
\left(\frac{1}{1-s z-z^{2}}\right)^{x}=\sum_{n=0}^{\infty} \mathcal{F}_{n+1}(s ; x) z^{n}
$$

and

$$
\left(\frac{1}{1-s z-t z^{2}}\right)^{x}=\sum_{n=0}^{\infty} \mathcal{F}_{n+1}(s, t ; x) z^{n} .
$$

Remark 6. The idea of this paper comes from the papers and preprints [7, 9, 15. 16, 22, 23, 24, 25, 26, 27, 28, 30, 31, 32, 35, 36, 37, 38, 39, 41, 43, 44, 45, 51, 52, 53, 54, 56, 57, 58.

Remark 7. This paper is an expanded and revised version of the preprint [20].

Acknowledgments. The author thanks Professor Taekyun Kim for his sending an electronic version of the paper [11] on 22 July 2016. The author thanks Professor Jeffrey O. Shallit for his careful corrections to the original version of this paper.

\section{REFERENCES}

[1] A. Aboud, J.-P. Bultel, A. Chouria, J.-G. Luque, O. Mallet, Bell polynomials in combinatorial Hopf algebras, arXiv preprint (2014), available online at http://arxiv.org/abs/1402.2960

[2] G. E. Bergum and V. E. Hoggatt Jr., Limits of quotients for the convolved Fibonacci sequence and related sequences, Fibonacci Quart. 15 (1977), 113-116.

[3] G. E. Bergum and V. E. Hoggatt Jr., Numerator polynomial coefficient array for the convolved Fibonacci sequence, Fibonacci Quart. 14 (1976), 43-48.

[4] L. Comtet, Advanced Combinatorics: The Art of Finite and Infinite Expansions, Revised and Enlarged Edition, D. Reidel Publishing Co., 1974; available online at https://doi.org/ 10.1007/978-94-010-2196-8

[5] H. W. Corley, The convolved Fibonacci equation, Fibonacci Quart. 27 (1989), 283-284.

[6] B.-N. Guo and F. Qi, An explicit formula for Bernoulli numbers in terms of Stirling numbers of the second kind, J. Anal. Number Theory 3 (2015), no. 1, 27-30.

[7] B.-N. Guo and F. Qi, Explicit formulae for computing Euler polynomials in terms of Stirling numbers of the second kind, J. Comput. Appl. Math. 272 (2014), 251-257; available online at https://doi.org/10.1016/j.cam.2014.05.018

[8] B.-N. Guo and F. Qi, Six proofs for an identity of the Lah numbers, Online J. Anal. Comb. 10 (2015), 5 pages.

[9] B.-N. Guo and F. Qi, Some identities and an explicit formula for Bernoulli and Stirling numbers, J. Comput. Appl. Math. 255 (2014), 568-579; available online at https://doi. org/10.1016/j.cam.2013.06.020

[10] B.-N. Guo and F. Qi, Some integral representations and properties of Lah numbers, J. Algebra Number Theory Acad. 4 (2014), no. 3, 77-87.

[11] T. Kim, D. V. Dolgy, D. S. Kim, and J. J. Seo, Convolved Fibonacci numbers and their applications, Ars Combin. 135 (2017), 119-131.

[12] P. Moree, Convoluted convolved Fibonacci numbers, J. Integer Seq. 7 (2004), Article 04.2.2, 14 pages.

[13] F. Qi, A new formula for the Bernoulli numbers of the second kind in terms of the Stirling numbers of the first kind, Publ. Inst. Math. (Beograd) (N.S.) 100(114) (2016), 243-249; available online at https://doi.org/10.2298/PIM150501028Q

[14] F. Qi, An explicit formula of a sequence of polynomials, IAENG Int. J. Appl. Math. 50 (2020), no. 2, in press.

[15] F. Qi, A simple form for coefficients in a family of nonlinear ordinary differential equations, Adv. Appl. Math. Sci. 17 (2018), no. 8, 555-561. 
[16] F. Qi, A simple form for coefficients in a family of ordinary differential equations related to the generating function of the Legendre polynomials, Adv. Appl. Math. Sci. 17 (2018), no. $11,693-700$.

[17] F. Qi, Derivatives of tangent function and tangent numbers, Appl. Math. Comput. 268 (2015), 844-858; available online at https://doi.org/10.1016/j.amc.2015.06.123

[18] F. Qi, Diagonal recurrence relations for the Stirling numbers of the first kind, Contrib. Discrete Math. 11 (2016), 22-30; available online at https://doi.org/10.11575/cdm.v11i1. 62389

[19] F. Qi, Explicit formulas for computing Bernoulli numbers of the second kind and Stirling numbers of the first kind, Filomat 28 (2014), 319-327; available online at https://doi.org/ 10.2298/FIL14023190

[20] F. Qi, Explicit formulas for the convolved Fibonacci numbers, ResearchGate Preprint (2016), available online at https://doi.org/10.13140/RG.2.2.36768.17927

[21] F. Qi, Integral representations and properties of Stirling numbers of the first kind, J. Number Theory 133 (2013), 2307-2319; available online at https://doi.org/10.1016/j.jnt.2012. 12.015

[22] F. Qi, Notes on several families of differential equations related to the generating function for the Bernoulli numbers of the second kind, Turkish J. Anal. Number Theory 6 (2018), no. 2, 40-42; available online at https://doi.org/10.12691/tjant-6-2-1

[23] F. Qi, Simple forms for coefficients in two families of ordinary differential equations, Glob. J. Math. Anal. 6 (2018), no. 1, 7-9; available online at https://doi.org/10.14419/gjma. v6i1.9778

[24] F. Qi, Simplification of coefficients in two families of nonlinear ordinary differential equations, Turkish J. Anal. Number Theory 6 (2018), no. 4, 116-119; available online at https://doi.org/10.12691/tjant-6-4-2

[25] F. Qi, Simplifying coefficients in a family of nonlinear ordinary differential equations, Acta Comment. Univ. Tartu. Math. 22 (2018), no. 2, 293-297; available online at https://doi. org/10.12697/ACUTM.2018.22.24

[26] F. Qi, Simplifying coefficients in a family of ordinary differential equations related to the generating function of the Laguerre polynomials, Appl. Appl. Math. 13 (2018), no. 2, 750-755.

[27] F. Qi, Simplifying coefficients in a family of ordinary differential equations related to the generating function of the Mittag-Leffler polynomials, Korean J. Math. 27 (2019), no. 2, 417-423; available online at https://doi.org/10.11568/kjm.2019.27.2.417.

[28] F. Qi, Simplifying coefficients in differential equations related to generating functions of reverse Bessel and partially degenerate Bell polynomials, Bol. Soc. Paran. Mat. 39 (2021), no. 4 , in press; available online at http://dx.doi.org/10.5269/bspm.41758

[29] F. Qi, V. Čerňanová, X.-T. Shi, and B.-N. Guo, Some properties of central Delannoy numbers, J. Comput. Appl. Math. 328 (2018), 101-115; available online at https://doi.org/10.1016/ j.cam.2017.07.013

[30] F. Qi and B.-N. Guo, A diagonal recurrence relation for the Stirling numbers of the first kind, Appl. Anal. Discrete Math. 12 (2018), no. 1, 153-165; available online at https://doi. org/10.2298/AADM170405004Q

[31] F. Qi and B.-N. Guo, Explicit formulas and recurrence relations for higher order Eulerian polynomials, Indag. Math. 28 (2017), no. 4, 884-891; available online at https://doi.org/ 10.1016/j.indag.2017.06.010

[32] F. Qi and B.-N. Guo, Explicit formulas for derangement numbers and their generating function, J. Nonlinear Funct. Anal. 2016, Article ID 45, 10 pages.

[33] F. Qi and B.-N. Guo, Explicit formulas for special values of the Bell polynomials of the second kind and for the Euler numbers and polynomials, Mediterr. J. Math. 14 (2017), no. 3, Article 140, 14 pages; available online at https://doi.org/10.1007/s00009-017-0939-1

[34] F. Qi and B.-N. Guo, Expressing the generalized Fibonacci polynomials in terms of a tridiagonal determinant, Matematiche (Catania) 72 (2017), no. 1, 167-175; available online at https://doi.org/10.4418/2017.72.1.13

[35] F. Qi and B.-N. Guo, Several explicit and recursive formulas for generalized Motzkin numbers, AIMS Math. 5 (2020), no. 2, 1333-1345; available online at https://doi.org/10.3934/math. 2020091

[36] F. Qi and B.-N. Guo, Some properties of the Hermite polynomials, Georgian Math. J. 27 (2020), in press; available online at https://doi.org/10.20944/preprints201611.0145.v1 
[37] F. Qi and B.-N. Guo, Viewing some ordinary differential equations from the angle of derivative polynomials, Iran. J. Math. Sci. Inform. 15 (2020), no. 2, in press; available online at https://doi.org/10.20944/preprints201610.0043.v1

[38] F. Qi, D. Lim, and B.-N. Guo, Explicit formulas and identities for the Bell polynomials and a sequence of polynomials applied to differential equations, Rev. R. Acad. Cienc. Exactas Fís. Nat. Ser. A Mat. RACSAM 113 (2019), no. 1, 1-9; available online at https://doi.org/10. 1007/s13398-017-0427-2

[39] F. Qi, D. Lim, and B.-N. Guo, Some identities related to Eulerian polynomials and involving the Stirling numbers, Appl. Anal. Discrete Math. 12 (2018), no. 2, 467-480; available online at https://doi.org/10.2298/AADM171008014Q

[40] F. Qi, D. Lim, and Y.-H. Yao, Notes on two kinds of special values for the Bell polynomials of the second kind, Miskolc Math. Notes 20 (2019), no. 1, 465-474; available online at https: //doi.org/10.18514/MMN.2019.2635

[41] F. Qi, A.-Q. Liu, and D. Lim, Explicit expressions related to degenerate Cauchy numbers and their generating function, In: J. Singh, D. Kumar, H. Dutta, D. Baleanu, and S. Purohit (eds), Mathematical Modelling, Applied Analysis and Computation, ICMMAAC 2018, Springer Proceedings in Mathematics \& Statistics, vol. 272, Chapter 2, pp. 41-52, Springer, Singapore, 2019; available online at https://doi.org/10.1007/978-981-13-9608-3_2

[42] F. Qi, P. Natalini, and P. E. Ricci, Recurrences of Stirling and Lah numbers via second kind Bell polynomials, Discrete Mathematics Letters 3 (2020), 31-36.

[43] F. Qi, D.-W. Niu, and B.-N. Guo, Simplification of coefficients in differential equations associated with higher order Frobenius-Euler numbers, Tatra Mt. Math. Publ. 72 (2018), 67-76; available online at https://doi.org/10.2478/tmmp-2018-0022.

[44] F. Qi, D.-W. Niu, and B.-N. Guo, Simplifying coefficients in differential equations associated with higher order Bernoulli numbers of the second kind, AIMS Math. 4 (2019), no. 2, 170-175; available online at https://doi.org/10.3934/Math.2019.2.170.

[45] F. Qi, D.-W. Niu, and B.-N. Guo, Some identities for a sequence of unnamed polynomials connected with the Bell polynomials, Rev. R. Acad. Cienc. Exactas Fís. Nat. Ser. A Math. RACSAM 113 (2019), no. 2, 557-567; available online at https://doi.org/10.1007/ s13398-018-0494-z

[46] F. Qi, D.-W. Niu, D. Lim, and B.-N. Guo, Closed formulas and identities for the Bell polynomials and falling factorials, Contrib. Discrete Math. 15 (2020), no. 1, 163-174; available online at https://doi.org/10.11575/cdm.v15i1.68111

[47] F. Qi, D.-W. Niu, D. Lim, and B.-N. Guo, Some properties and an application of multivariate exponential polynomials, Math. Methods Appl. Sci. 43 (2020), no. 6, 2967-2983; available online at https://doi.org/10.1002/mma.6095.

[48] F. Qi, D.-W. Niu, D. Lim, and Y.-H. Yao, Special values of the Bell polynomials of the second kind for some sequences and functions, J. Math. Anal. Appl. ??? (2020), no. ?, in press; available online at https://doi.org/10.1016/j.jmaa.2020.??.???

[49] F. Qi, X.-T. Shi, and F.-F. Liu, Several identities involving the falling and rising factorials and the Cauchy, Lah, and Stirling numbers, Acta Univ. Sapientiae Math. 8 (2016), no. 2, 282-297; available online at https://doi.org/10.1515/ausm-2016-0019

[50] F. Qi, X.-T. Shi, F.-F. Liu, and D. V. Kruchinin, Several formulas for special values of the Bell polynomials of the second kind and applications, J. Appl. Anal. Comput. 7 (2017), no. 3, 857-871; available online at https://doi.org/10.11948/2017054

[51] F. Qi, J.-L. Wang, and B.-N. Guo, Notes on a family of inhomogeneous linear ordinary differential equations, Adv. Appl. Math. Sci. 17 (2018), no. 4, 361-368.

[52] F. Qi, J.-L. Wang, and B.-N. Guo, Simplifying and finding ordinary differential equations in terms of the Stirling numbers, Korean J. Math. 26 (2018), no. 4, 675-681; available online at https://doi.org/10.11568/kjm.2018.26.4.675

[53] F. Qi, J.-L. Wang, and B.-N. Guo, Simplifying differential equations concerning degenerate Bernoulli and Euler numbers, Trans. A. Razmadze Math. Inst. 172 (2018), no. 1, 90-94; available online at https://doi.org/10.1016/j.trmi.2017.08.001

[54] F. Qi and Y.-H. Yao, Simplifying coefficients in differential equations for generating function of Catalan numbers, J. Taibah Univ. Sci. 13 (2019), no. 1, 947-950; available online at https://doi.org/10.1080/16583655.2019.1663782 
[55] F. Qi and M.-M. Zheng, Explicit expressions for a family of the Bell polynomials and applications, Appl. Math. Comput. 258 (2015), 597-607; available online at https://doi.org/ $10.1016 / \mathrm{j}$.amc.2015.02.027

[56] F. Qi and J.-L. Zhao, Some properties of the Bernoulli numbers of the second kind and their generating function, Bull. Korean Math. Soc. 55 (2018), no. 6, 1909-1920; available online at https://doi.org/10.4134/BKMS.b180039

[57] F. Qi, Q. Zou, and B.-N. Guo, The inverse of a triangular matrix and several identities of the Catalan numbers, Appl. Anal. Discrete Math. 13 (2019), no. 2, 518-541; available online at https://doi.org/10.2298/AADM190118018Q

[58] J.-L. Zhao, J.-L. Wang, and F. Qi, Derivative polynomials of a function related to the ApostolEuler and Frobenius-Euler numbers, J. Nonlinear Sci. Appl. 10 (2017), no. 4, 1345-1349; available online at https://doi.org/10.22436/jnsa.010.04.06

Institute of Mathematics, Henan Polytechnic University, Jiaozuo 454010, Henan, China; College of Mathematics and Physics, Inner Mongolia University for Nationalities, Tongliao 028043, Inner Mongolia, China; School of Mathematical Sciences, Tianjin Polytechnic University, Tianjin 300387, China

Email address: qifeng618@gmail.com, qifeng618@hotmail.com, qifeng618@qq.com

$U R L:$ https://qifeng618. wordpress.com 